\title{
Diagnostik und Therapie der Harnleitersteinkolik
}

\author{
Thomas H. Forster ${ }^{1,2}$, Gernot Bonkat ${ }^{2}$, Stephen Wyler ${ }^{2}$, Robin Ruszat ${ }^{2}$, Nicole Ebinger ${ }^{2}$, \\ Thomas C. Gasser ${ }^{2}$ und Alexander Bachmann ${ }^{2}$ \\ ${ }^{1}$ Urologische Klinik, Solothurner Spitäler AG (soH), Schweiz \\ ${ }^{2}$ Urologische Universitätsklinik beider Basel, Schweiz
}

Eingegangen am 13. September 2007, angenommen nach Revision am 16. April 2008

(C) Springer-Verlag 2008

\section{Diagnosis and therapy of acute ureteral colic}

Summary. Acute ureteral colic presents with a complex of acute and characteristic flank pain that usually indicates the presence of a stone in the urinary tract. Diagnosis and management of renal colic have undergone considerable evolution and advancement in recent years. The application of noncontrast helical computed tomography (CT) in patients with suspected ureteral colic is one major advance in the primary diagnostic process. The superior sensitivity and specificity of helical CT allow ureterolithiasis to be diagnosed without the potential side effects of contrast media. Initial management is based on three key concepts: $(A)$ rational and fast diagnostic process (B) effective pain control (C) and understanding of the impact of stone location and size on the natural course of the disease and definitive urologic management. These concepts are discussed in this review with reference to contemporary literature.

Key words: Renal colic, ureteral stone, watch and wait, ureterorenoscopy, shockwave lithotripsy.

Zusammenfassung. Die akute Harnleiterkolik präsentiert sich mit charakteristischen, kolikartigen Flankenschmerzen. Diese sind in der Regel für ein Konkrement im Bereich der ableitenden Harnwege hinweisend. Diagnostik und Therapie der Ureterkolik haben in den letzten Jahren eine intensive Veränderung und Weiterentwicklung erfahren. Eine große Verbesserung in der Primärdiagnostik stellt die Anwendung der Nativcomputertomographie dar. Durch überlegene Sensitivität und Spezifität dieser Technik ist die schnelle und definitive Diagnostik möglich. Potentielle Nebenwirkungen des Kontrastmittels sind hierbei ausgeschlossen. Das Management einer Harnleiterkolik basiert auf den drei Säulen (A) rationale und schnelle Diagnostik, (B) effektive Schmerzbekämpfung und (C) Kenntnisse über den natürlichen Krankheitsverlauf sowie die definitive urologische Therapie aufgrund der Konkrementgröße und Lokalisation. Diese Konzepte werden in dieser Arbeit mit Bezug auf aktuelle Literatur diskutiert.
Schlüsselwörter: Nierenkolik, Harnleiterstein, watch and wait, Ureterorenoskopie, Stoßwellentherapie.

\section{Einleitung}

Bedingt durch stärkste Schmerzen ist die Harnleitersteinkolik für den Patienten sehr bedrohlich und führt meist zu Notfallkonsultationen beim Hausarzt oder im Spital. Das Harnsteinleiden (Urolithiasis) ist weltweit ein Problem. In Ländern mit hohem sozioökonomischem Status ist die Inzidenz der Urolithiasis im Anstieg begriffen. So stieg von 1979 bis 2001 in Deutschland die Inzidenz des Harnsteinleidens um fast ein Prozent von 0,54 auf $1,47 \%$. Im gleichen Zeitraum erhöhte sich die Prävalenz von $4 \%$ auf 4,7\% [1]. Als mögliche Gründe hierfür werden unter anderem eine verbesserte und flächendeckend angebotene bildgebende Diagnostik sowie veränderte Ernährungsgewohnheiten diskutiert. Der Häufigkeitsgipfel der Erkrankung liegt zwischen dem 20. und 40. Lebensjahr [1]. Viele betroffene Patienten stehen im Erwerbsleben und der durch die Krankheit verursachte sozioökonomische Schaden ist beträchtlich. In den USA wurden die durch Nierensteinerkrankungen verursachten Kosten durch Arbeitsunfähigkeit 1993 auf US\$ 139 Millionen geschätzt. Zur Behandlung von Nierensteinen wurde 1.23 Milliarden US-Dollar aufgewendet (ca. 4,5 US\$ pro Einwohner) [2]. Eine schnelle und adäquate Diagnostik und Therapie der Harnleiterkolik ist somit nicht nur aus medizinischen, sondern auch aus sozioökonomischer Sicht zu fordern. In diesem Sinne soll diese Arbeit eine Übersicht über das Thema geben, wobei besonderer Wert auf die Einarbeitung der aktualisierten Leitlinien der Europäischen und Amerikanischen Gesellschaft für Urologie (European Association of Urology (EAU), American Urologic Association (AUA)) gelegt wurde [3]. Nach einem Kapitel zur Diagnostik der Harnleitersteinkolik folgen Ausführungen zur Pathophysiologie. Um die Lesbarkeit des Abschnittes „Therapie der Harnleitersteinkolik“ zu erleichtern, folgt sie dem im klinischen Alltag praktizierten ,erst die Kolikschmerzen behandeln, dann den Stein therapieren" und ist in zwei Teile gegliedert. Der erste Teil 
befasst sich mit der Behandlung der Harnleitersteinkolik selbst, der zweite Teil erläutert die Therapie des Harnleitersteines.

\section{Klinik und Diagnostik}

In den meisten Fällen wird die Urolithiasis symptomatisch, wenn ein Konkrement von der Niere in den Ureter gelangt und den Harnfluss obstruiert. Die Verdachtsdiagnose Harnleitersteinkolik basiert auf dem typischen Schmerzbild und wird durch Urinuntersuchung und Bildgebung bestätigt.

\section{Klinik}

Kolikartige Flankenschmerzen mit Ausstrahlung in den Unterbauch, die Leiste und das äußere Genitale verbunden mit vegetativen Begleiterscheinungen wie Übelkeit und Erbrechen sind typisch für einen Harnleiterstein. Die Patienten sind unruhig. Sie finden keine Position, in der der Schmerz erträglicher wird. In Abhängigkeit von der Steinlokalisation projiziert sich der Kolikschmerz in die Flanke oder den Unterbauch (proximaler und mittlerer Harnleiter) sowie das äußere Genitale (distaler Harnleiter). Flankenschmerzen sind weiterhin Ausdruck einer Nierenkapselspannung. Bei unmittelbar prävesikaler Lokalisation des Konkrementes beklagen viele Patienten Pollakisurie und Blasentenesmen.

\section{Urinuntersuchung}

Eine isolierte Mikrohämaturie ist ein typischer Befund bei der Urinuntersuchung. In bis zu $15 \%$ der Fälle lässt sich aber keine Mikrohämaturie feststellen [4].

\section{Bildgebung}

Sowohl die Computertomographie (CT) als auch das Intravenöse Urogramm (IVU) lassen eine Beurteilung der Lokalisation und Größe des Harnleiterkonkrementes zu. Diese Informationen sind zur Therapieplanung wichtig.

Die Untersuchung der ersten Wahl bei einer Harnleiterkolik ist die Nativ-Spiral-Computertomographie (siehe Abb. 1) [3]. Sie findet Harnleitersteine mit einer hohen Sensitivität (94-96\%) und Spezifität (94-100\%) unabhängig von Steingröße, Lokalisation und Steinzusammensetzung $[5,6]$. Im Vergleich zum Abdomen-Leerbild wird die Steingröße durch die rechnerische Rekonstruktion im cranio-caudalen Durchmesser häufig um bis zu 1,4 mm überschätzt [7]. Die CT kann sofort und ohne Darmvorbereitung durchgeführt werden. Sie ist dem Intravenösen Urogramm (IVU) insbesondere bei der Diagnose kleiner Steine überlegen und kann unabhängig von der Nierenfunktion durchgeführt werden [8]. Zudem können computertomographisch sekundäre Zeichen einer Ureterobstruktion nachgewiesen werden (siehe Tabelle 1). Diese persistieren auch für eine gewisse Zeit nach einem spontanen

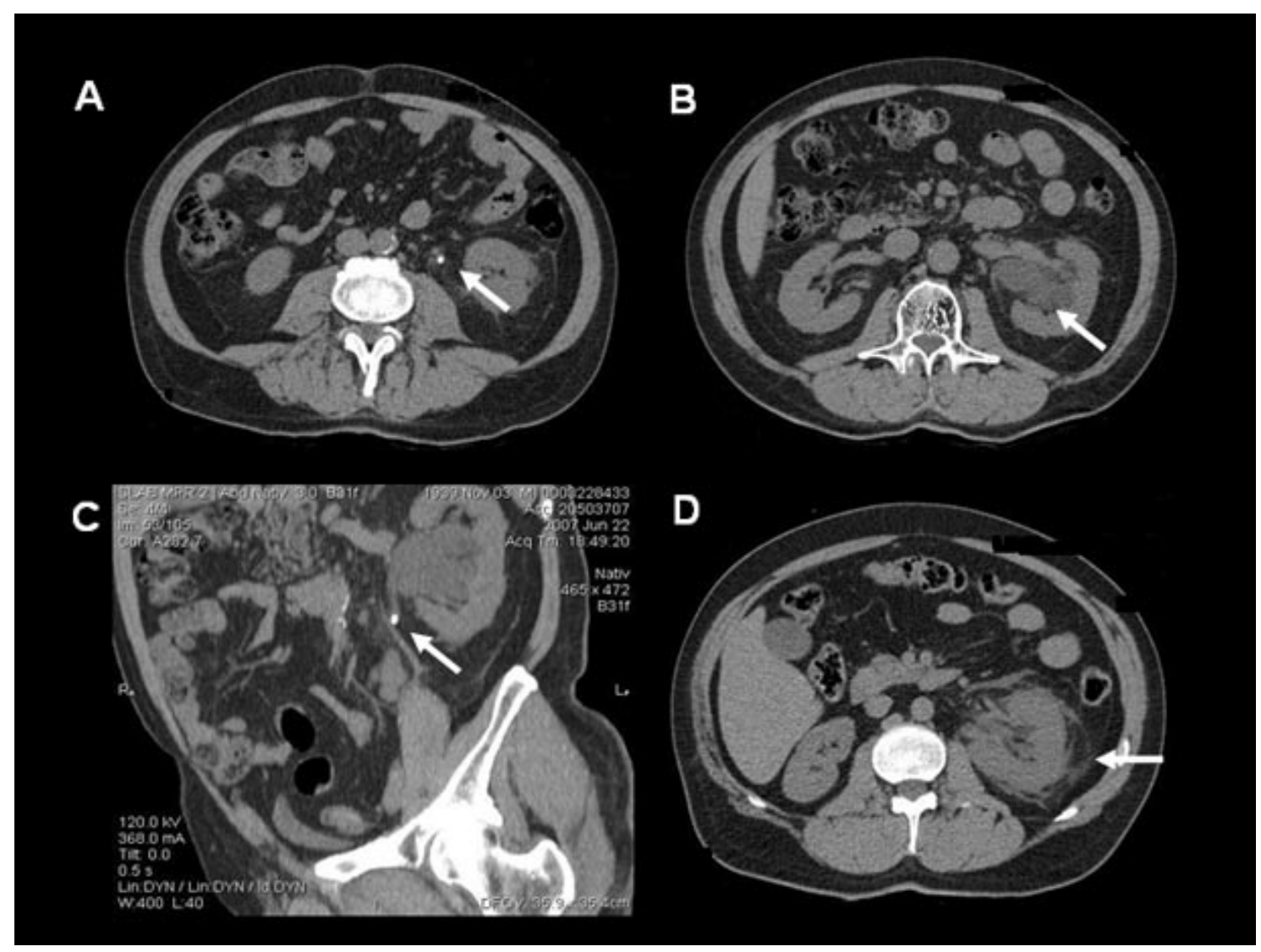

Abb. 1. Steindiagnostik im Computertomogramm. A Harnleiterstein auf Höhe des Nierenunterpols mit ausgeprägtem, periureteralem Stranding (Pfeil). B Gleicher Patient wie A: Konsekutive Harnstauungsniere (Pfeil). C Gleicher Patient wie A: sagitale Rekonstruktion (Pfeil zeigt auf den Stein). D Ausgeprägtes perirenales Stranding mit Verdickung der Gerota'schen Faszie (Pfeil) 


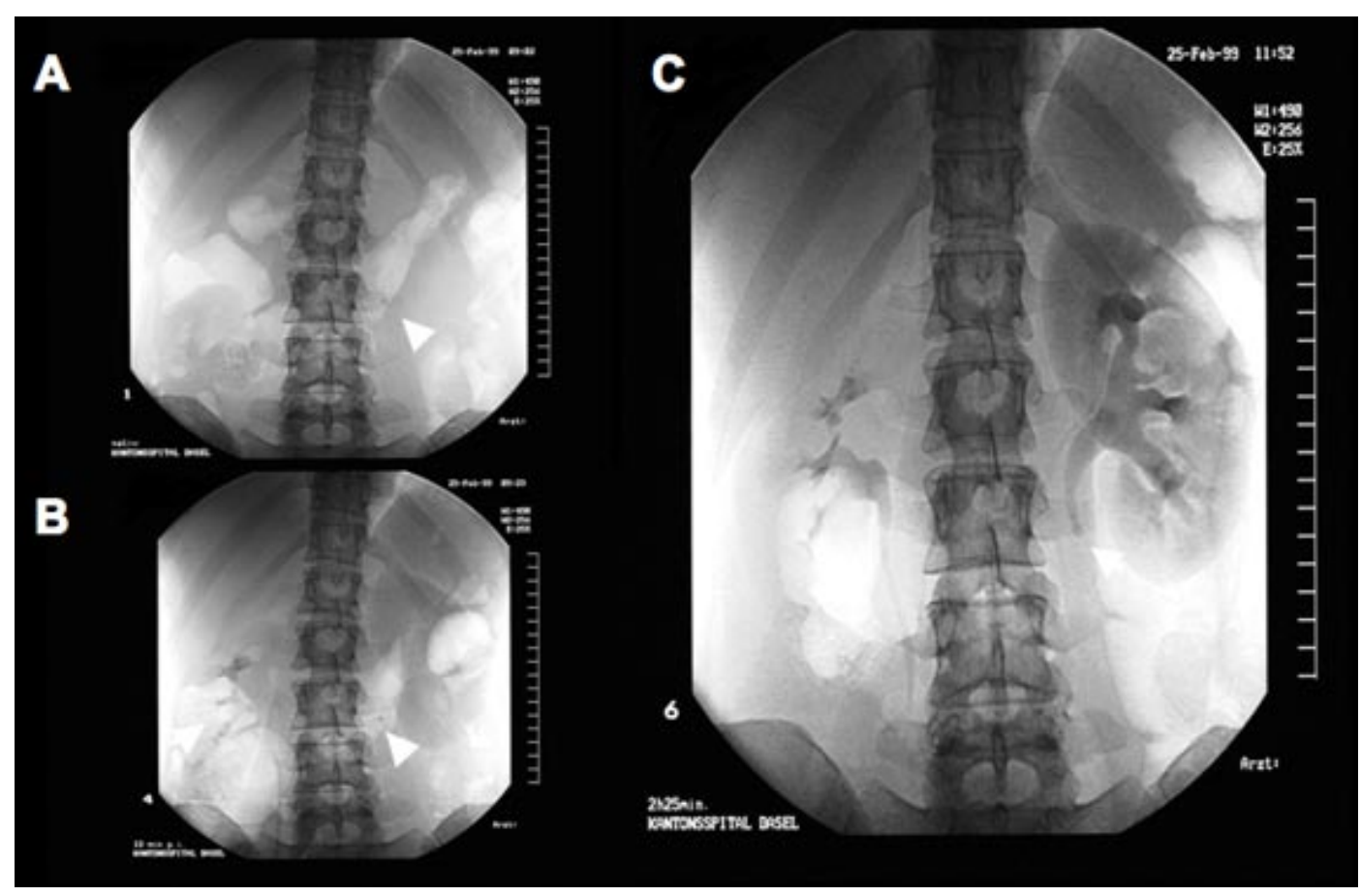

Abb. 2. Steindiagnostik im Ausscheidungsurogramm. A Abdomen leer Übersichtsaufnahme: Ausschnitt (Pfeildreieck zeigt auf den Stein). B Regelrechte Kontrastmittelausscheidung 5 Minuten post injectionem rechts. Fehlender nephrographischer und urographischer Effekt links (Pfeildreieck zeigt auf den Stein). C Verzögerter nephrographischer und urographischer Effekt links $2 \frac{1}{2} \mathrm{~h}$ post injectionem. Kontrastmittelstop am proximalem Harnleiterstein

Steinabgang und können so auf diesen hinweisen [9]. Bei fehlendem Steinnachweis liefert die CT oft wegweisende Befunde für die weitere Abklärung der Schmerzursache sowie bis dato unbekannte Pathologien [6]. Ein häufig genannter Nachteil der Nativ-CT ist die bis 3 Mal erhöhte Strahlenbelastung im Vergleich zum IVU [6, 10, 11]. Die Entwicklung von Low-dose- und Ultra-low-dose-Untersuchungsprotokollen führte zu vergleichbaren Steindetektionsraten bei einer Strahlenexposition die zum Teil unterhalb der des IVU liegen [10, 12, 13].

Ist die Durchführung einer CT in der Notfallsituation nicht möglich, so kann im schmerzfreien Intervall ein Intravenöses Urogramm (IVU) durchgeführt werden (siehe Abb. 2). Dieses hat ebenfalls eine hohe diagnostische Sicherheit (Sensitivität: 85-87\%; Spezifität: 90-94\%) [5]. Die Kontrastmittelausscheidung der betroffenen Niere im Vergleich zur gesunden Seite lässt Rückschlüsse auf die

Tabelle 1. Sekundäre Zeichen einer Ureterobstruktion [9]

Hydronephrose

(Dilatation Nierenbeckenkelchsystem)

Einseitige Vergrößerung der Niere

Perinephritisches Ödem (Stranding)

Hydroureter (Dilatation des Harnleiters)

Periureterales Ödem (Stranding)

Verdickung der perirenalen Faszie (Gerota'sche Faszie)
Nierenfunktion und die Obstruktion der ableitenden Harnwege zu. Es besteht keine Interferenz mit metallenen Implantaten wie z.B. Hüftgelenksprothesen. Komplikationen wie die kontrastmittelinduzierte Exazerbation der Kolik (osmotische Diurese), Kelchrupturen und allergische Reaktionen treten nur beim IVU auf [8].

Die Sonographie kann Harnleitersteine am pyeloureteralen Übergang und juxtavesikal bei gefüllter Harnblase darstellen (siehe Abb. 3). Entlang des Ureterverlaufes ist

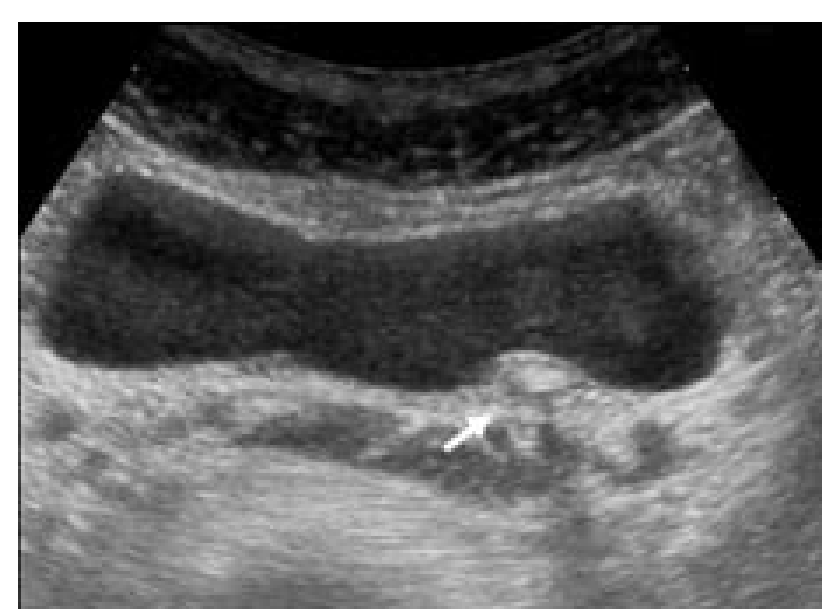

Abb. 3. Sonographischer Nachweis eines $5 \mathrm{~mm}$ messenden intramuralen Konkrementes links mit charakteristischem dorsalem Schallschatten (Pfeil zeigt auf den Stein) 
der Konkrementnachweis sonographisch oft schwierig. Gründe hierfür sind unter anderem Darmgasüberlagerungen und die tiefe retroperitoneale Lage des Ureters. Findet sich in der Ultraschalluntersuchung eine Dilatation des Nierenbeckenkelchsystemes oder ein fehlender Urin-Jet, erhärtet dies die Verdachtsdiagnose ,Harnleiterstein'. Eine Dilatation des Nierenbeckenkelchsystems kann mittels Ultraschall gleich sicher diagnostiziert werden wie im Nativ-CT (Sensitivität: 72-100\%; Spezifität: 59-100\%). Weiterhin ist zu bedenken, dass eine fehlende Erweiterung des NBKS eine akute Obstruktion innerhalb der ersten 12-24 Stunden keineswegs ausschließt. Diese manifestiert sich in einigen Fällen erst im Laufe von Stunden bis Tagen [14]. Hinsichtlich präziser Steindiagnostik (Lokalisation, Größe, Anzahl) ist der Ultraschall der CT aber deutlich unterlegen $[8,15]$. Wegen der mit der CT und dem IVU verbundenen Strahlenbelastung ist die Sonographie Untersuchung der Wahl bei Schwangeren und Kindern [14].

Die Aussagekraft des konventionellen AbdomenLeerbildes im Liegen alleine ist eingeschränkt. Konkremente sind erst ab einer Größe von 3-4 mm zu diagnostizieren. Die Sensitivität liegt bei 45-60\%. Gründe hierfür sind unter anderem Darmgas- und Stuhlüberlagerungen sowie eine fallabhängige schwierige Differenzierung von abdominellen oder pelvinen Verkalkungen (Phlebolithen, verkalkte mesenteriale Lymphknoten). Weiterhin sind 10-20\% der Steine röntgen-negativ (Harnsäuresteine, Zystinsteine sind nur schwach röntgen-positiv) [16].

Abdomen-Leerbild und Sonographie spielen im Rahmen der Notfalldiagnostik in der Hand geübter Untersucher eine wichtige Rolle und erreichen eine hohe diagnostische Sicherheit (93\% Sensitivität und $95 \%$ Spezifität) $(17,18]$. Harnleitersteine, die so nicht erkannt werden, sind oft klein $(<5 \mathrm{~mm})$ und im mittleren oder unteren Harnleiter lokalisiert und damit meist spontan abgangsfähig [19].

Abdomen-Leerbild und Sonographie können als primäres Diagnostikum überall dort empfohlen werden, wo eine CT nicht unmittelbar verfügbar ist.

\section{Pathophysiologie der Harnleitersteinkolik}

Durch die partielle oder vollständige Obstruktion des Harnleiters durch den Stein kommt es zur Erhöhung des intraluminalen Drucks proximal des Konkrementes im Harnleiter und im Nierenbeckenkelchsystemes. Die Dehnung des Hohlraumsystemes führt zu Reizung von Nozizeptoren, welche im Bereich der Submukosa und der Lamina propria der Nierenkapsel, des Nierenbeckens und des Ureters liegen. Als Mechanorezeptoren reagieren sie auf Dehnung stärker, als z.B. auf die direkte Irritation durch einen scharfen oder spitzen Stein. Es kommt zu einer Ausschüttung von Prostaglandin E2 (PgE2), welches das Schwellenpotential der Nozizeption senkt, zu einer Erhöhung der glomerulären Filtrationsrate (GFR) führt und eine Entzündungsreaktion auslöst [20]. Diese PgE2 assoziierten Phänomene verstärken den Schmerz. Über A- $\delta$-Fasern (myelinisiert und schnell leitend; $12-30 \mathrm{~m} / \mathrm{s}$, verantwortlich für scharf und stechenden Schmerz) und C-Fasern (nicht-myelinisiert, langsam leitend; $1 \mathrm{~m} / \mathrm{s}$, verantwortlich für dumpfen Dauerschmerz) werden die Schmerzimpulse zum Hinterhorn des Rückenmarkes weitergeleitet. Von hier aus werden sie nach Umschaltung über den Tractus spinothalamicus an supraspinale Schmerz-Zentren fortgeleitet werden. Die kolikassoziierten Symptome wie Übelkeit, Erbrechen, Tachykardie oder Abnahme der Peristaltik sowie die Projektion des Schmerzes in bestimmte Hautareale (Head'sche Zonen), bzw. in die Dermatome T10 bis S4 entstehen durch Umschaltung der Schmerzimpulse im Hinterhorn des Rückenmarkes auf Interneurone. Diese stimulieren efferente Neurone im Vorderhorn. Die Hyperperistaltik des Harnleiters als Schmerzursache spielt im Vergleich zu den PgE2 vermittelten Schmerzen eine untergeordnete Rolle [21].

\section{Therapie der Harnleitersteinkolik}

Die Therapie der Harnleiterkolik erfolgt in zwei Phasen:

1. Schmerztherapie mit Prävention und/oder Therapie von Komplikationen

2. Steintherapie mit dem Ziel der Steinfreiheit.

\section{Medikamentöse Therapie}

Entsprechend der Pathophysiologie des NierenkolikSchmerzes sind nichtsteroidale antientzündliche Agenzien (NSAIA) und Metamizol die Therapeutika der ersten Wahl (siehe Tabelle 2). Parenteral verabreichte NSAIA sind gleich wirksam wie Opiate [22, 23]. Eine randomisierte, doppelblinde Multizenter-Studie der Collaborative Group of the Spanish Society of Clinical Pharmacology verglich die Wirksamkeit von Metamizol (1 g vs. $2 \mathrm{~g}$ i.m.), Diclofenac (75 mg i.m.) und Pethidin (100 mg i.m.), ohne dass sich ein Unterschied nachweisen ließ. Patienten unter Pethidin weisen aber mehr Nebenwirkungen (insb. Schwindel, Mundtrockenheit, Übelkeit, Erbrechen, orthostatische Hypotension, Schläfrigkeit und Schwitzen) auf [24]. Morphin und Pethidin sind gleich wirksam bezüglich Schmerzen; Pethidin hat aber das etwas schlechtere Nebenwirkungsprofil [25]. Metamizol i.m. und i.v. zeigten keine signifikanten Unterschiede in der Wirksamkeit [25]. Neben der i.m.- und der i.v.-Medikation von NSAIA ist die Wirksamkeit dieser Medikamente auch bei rektaler und sublingualer Verabreichungsform belegt [26, 27]. Die rektale Verabreichung (Indometacin $100 \mathrm{mg}$ rektal vs. $50 \mathrm{mg}$ i.v.) scheint aber weniger effektiv zu sei [28]. Sublingual appliziertes Piroxicam $(40 \mathrm{mg})$ ist gleich wirksam wie Diclofenac $75 \mathrm{mg}$ i.m. [27]. Bei bekannter, vorbestehender Niereninsuffizienz sollten NSAIA in der akuten Kolik nur zurückhaltend verabreicht werden, da sie die glomeruläre Filtrationsrate zusätzlich verschlechtern können. Die European Association of Urology (EAU) empfiehlt wenn immer möglich, die Schmerzbehandlung mit Diclofenac zu beginnen [3, 29].

Substanzen wie N-Butylscopolamin aus der Gruppe der Parasympatholytika werden aktuell in der Therapie der Harnleitersteinkolik nicht mehr empfohlen. Sie wirken nur im distalen parasympatisch innervierten Harnleiter. Um einen Effekt auf die glatte Muskulatur des distalen Harnleiters zu erreichen, müssen die Dosen sehr hoch gewählt werden. Hierbei treten unweigerlich Nebenwir- 
Tabelle 2. Medikamentöse Therapie der akuten Nierenkolik (Zusammenfassung)

- Therapie der 1. Wahl ist die Verabreichung eines schnellwirksamen Nicht-Opiates, z.B. (CAVE: Niereninsuffizienz):

- Diclofenac 75 mg i.m. (= Empfehlung der EAU)

- Metamizol 1 Amp. (2 ml) à $1000 \mathrm{mg}$ i.v. oder i.m. bis 4x/d

- Ketorolac 1 Amp. (1 ml) $30 \mathrm{mg}$ bis $3 \times / \mathrm{d}$ für max. 2 Tage

- Piroxicam $2 \times 20 \mathrm{mg}$ s.l. $1 \times / \mathrm{d}$

- Therapie der 2. Wahl, resp. Reservemedikation ist die Verabreichung eines Opiates, z.B.:

- Morphin 0,1 mg/kgKG i.m. oder 50-70\% s.c. $+30-50 \%$ i.v. bis $6 \times 1 /$ oder

$5 \mathrm{mg}$ i.v. initial gefolgt von wiederholt $2 \mathrm{mg}$ i.v. bis zur Schmerzfreiheit

- Pethidin $1 \mathrm{mg} / \mathrm{kgKG}$ i.m. oder $50-70 \%$ s.c. $+30-50 \%$ i.v. bis $6 \times 1 / \mathrm{d}$

- $\quad$ im Rahmen der Ersttherapie auf einer Notfallstation wird bei vorhandener Infusion eine i.v. Medikamentenapplikation wegen der genaueren Steuerbarkeit und dem schnellen Wirkungseintritt bevorzugt

- $\quad$ eine Schmerzprophylaxe mit Diclofenac Tbl. à 50 mg (1-1-1) p.o. kann erfolgen, sofern in den nächsten 48h keine ESWL geplant ist und die Nierenfunktion gut ist

- eine alpha-Blocker Therapie z.B. mit Tamsulosin 400ng einmal pro Tag kann bei distalen Steinen den Steinabgang beschleunigen

- $\quad$ keine forcierte Diurese

kungen auf (Tachykardie, Darmparalyse). Die auch heute noch in manchen Lehrbüchern vertretene Meinung, dass N-Butylscopolamin $\left(\right.$ Buscopan $^{\circledR}$ ) im Rahmen der Therapie der Nierenkolik einen nützlichen Effekt besitzt, liegt möglicherweise daran, dass früher mit Buscopan compositum $^{\circledR}$ ein wirksames Kombinationspräparat vorlag. Dieses enthielt Metamizol.

Eine forcierte Diurese (Infusionstherapie $>21 /$ d) führt zu einer zusätzlichen Dilatation des Nierenbeckenkelchsystemes und des Harnleiters, was zu einer Verstärkung der Schmerzen führen kann. Im dilatierten Harnleiter ist die Kontraktionskraft der Muskulatur gemindert, was den Weitertransport des Konkrementes erschwert. Eine forcierte Diurese ist deshalb kontraindiziert.

\section{Interventionelle Therapie: Doppel-J-Katheter Einlage und Nephrostomie}

Unter interventioneller Therapie versteht man die Ableitung der betroffenen Niere mittels Doppel-J-Katheter (Harnleiterschiene) oder perkutaner Nephrostomie (Nierenfistelkatheter). Indikationen zur interventionellen Therapie bei Nierenkolik sind Harnwegsinfektion mit drohender Urosepsis, rezidivierende therapierefraktäre Koliken und funktionelle Einnierigkeit mit Stauung. Die Ableitung des Harnsystemes kann mittels Doppel-J-Katheter oder perkutaner Nephrostomie erfolgen (siehe Abb. 4). Dies ist abhängig von Erfahrung und Können des behandelnden Urologen und der vorhandenen Infra-

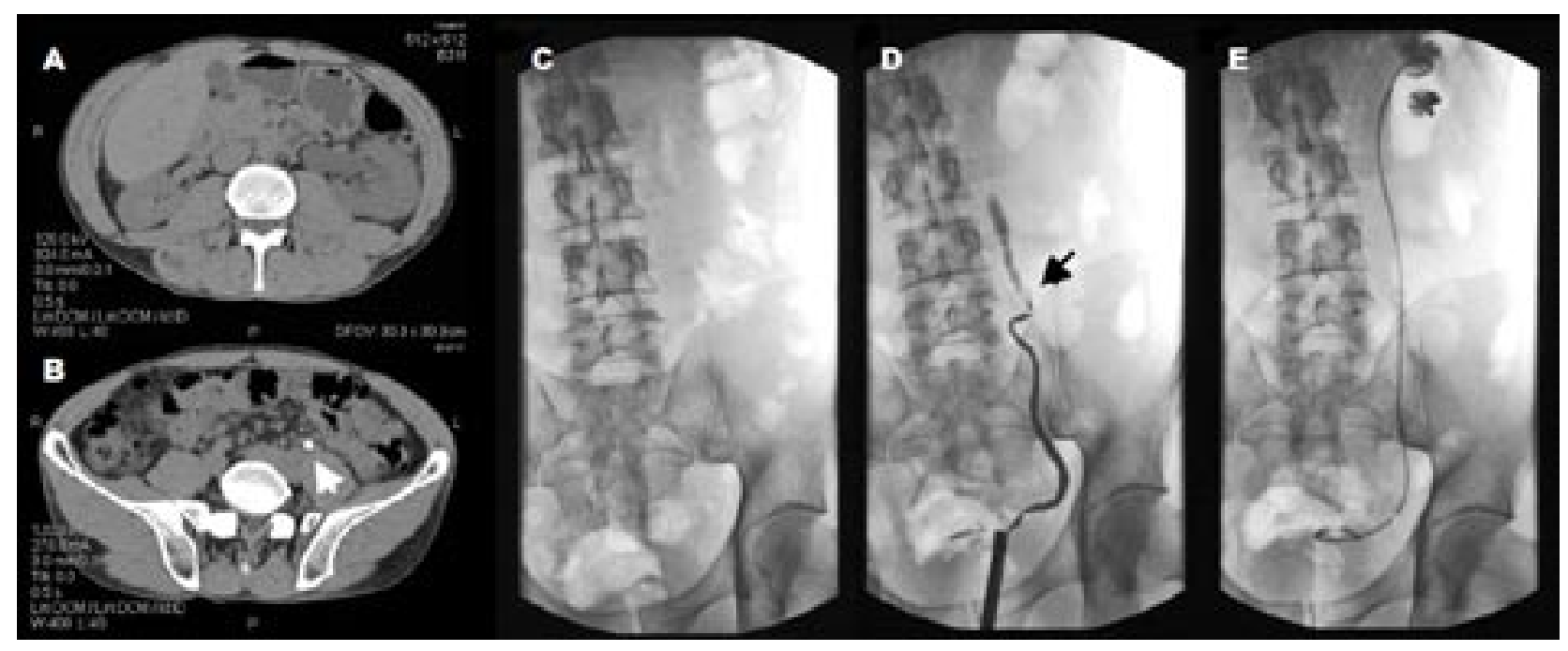

Abb. 4. Bei diesem 45-jährigen Patienten wurde auswärts wegen Flankenkoliken links eine Computertomographie durchgeführt. Als zusätzlich Fieber und Schüttelfrost auftraten wurde der Patient notfallmäßig zur weiteren Behandlung an die Urologische Klinik verlegt. A Harntransportstörung links mit Dilatation im Computertomogramm. B Nachweis eines mittleren Harnleitersteines links (Pfeil) im Computertomogramm. Einlage einer Harnleiterschiene (Doppel-J-Katheter) bei demselben Patienten aufgrund obstruktiver Pyelonephritis mit drohender Urosepsis. C Leerbild ohne Steinnachweis (Harnsäurestein!). D Retrogrades Pyelogramm: Deutlich von Kontrastmittel umspülter Harnleiterstein (Pfeil). E Liegender Doppel-J-Katheter 
Tabelle 3. Watchful-waiting (WW) bei Harnleitersteinen (Zusammenfassung)

Indikation: Harnleitersteine $\leq 5 \mathrm{~mm}$; Harnleitersteine $5-10 \mathrm{~mm}$ auf Wunsch des Patienten

Dauer: in der Regel nicht länger als 4 Wochen

Kontrolle der Steinlokalisation nach 10-14 Tagen und nach 4 Wochen (Röntgen, Sonographie, evt. CT)

Diclofenac $50 \mathrm{mg} 3 \times / \mathrm{d}$ zur Schmerzprophylaxe, evt. AlphaBlocker bei juxtavesikalen Steinen

Kontraindikationen zum watchful-waiting:

- Harnwegsinfektion

- $\quad$ rezidivierende, schlecht therapierbare Koliken

- Nierenfunktionsstörung

- funktionelle Einnierigkeit

- Nierenstauung bds.

- $\quad$ sozioökonomische Gründe, resp. Patientenwunsch

struktur [30]. Bei großen Harnleitersteinen, ausgeprägter Harnstauung, Infekt, jungen Männern, und evt. fehlender Narkosemöglichkeit wird eine Nephrostomie von gewissen Autoren empfohlen [31]. Andere Studien zeigen eine Überlegenheit des Doppel-J-Katheters [32]. Sofern kein Infekt vorliegt kann bei entsprechenden personellen und infrastrukturellen Möglichkeiten auch die direkte Steintherapie erfolgen.

\section{Therapie des Harnleitersteines}

Watchful-waiting - Spontanabgang

In Abhängigkeit der Steingröße und der Steinlokalisation sind bis zu 95\% der Harnleitersteine spontan abgangsfähig (siehe Tabelle 3). Bei Harnleitersteinen $>5 \mathrm{~mm}$ ist mit einem Spontanabgang in nur 50\% zu rechnen [33, 34].

Die Zeit von der Steindiagnose bis zum Steinabgang ist abhängig von der Steingröße und der Steinlokalisation (siehe Tabelle 4). Die durchschnittliche Dauer bis zum Steinabgang beträgt 8,12 und 22 Tage für Steine der Größe $\leq 2 \mathrm{~mm}, 2-4 \mathrm{~mm}$ und 4-6 mm. 95\% der Steine sind nach 31, 40 und 39 Tagen abgegangen [34].

Eine vereinfachte Berechnungsformel für die durchschnittliche Zeit bis zum Steinabgang ist (modifiziert nach 34):

Zeit $=10+5 \times$ Steingröße $(\mathrm{mm})-4 \times$ Steinlokalisation $($ proximal $=3$, Mitte $=2$, distal $=1$ )
Ein watchful-waiting (WW) ist bei Harnleitersteinen sinnvoll, die kleiner oder gleich $5 \mathrm{~mm}$ sind. Sollte der Patient ein abwartendes Vorgehen ausdrücklich wünschen, kann auch bei Harnleitersteinen von $5-10 \mathrm{~mm}$ Durchmesser vorerst zugewartet werden. Der Patient sollte aber über die geringe Chance eines spontanen Steinabganges informiert werden. Kontraindikationen für WW sind eine Harnwegsinfektion, rezidivierende, schwer zu therapierende Koliken, Niereninsuffizienz, funktionelle Einnierigkeit oder eine beidseitige Nierenstauung. Ein abwartendes Vorgehen von mehr als 4 Wochen ist problematisch, da bei länger dauernder Obstruktion (>4 Wochen) das Risiko einer irreversiblen Nierenschädigung steigt $[35,36]$. Diclofenac p.o. kann als Prophylaxe das Auftreten neuer Koliken und die damit zusammenhängenden Re-Hospitalisationen reduzieren. Die Rate der Spontanabgänge kann aber nicht beeinflusst werden [37]. In unseren Händen hat sich die Therapie mit Diclofenac $50 \mathrm{mg} 3 \times / \mathrm{d}$ ergänzt durch Metamizol $1 \mathrm{~g}$ als Suppositorium bis $4 \times / d$ in Reserve bewährt. NSAIA sind potentiell nephrotoxisch und sollten bei Patienten mit vorbestehender Niereninsuffizienz nicht eingesetzt werden (Kontraindikation). Daneben kommt es $\mathrm{zu}$ einer reversiblen Thrombozytenfunktionsstörung, was vor einer geplanten extracorporalen Stoßwellenilthotripsie (ESWL) in Betracht gezogen werden muss. Alpha-Rezeptorblocker, welche zur Therapie der benignen Prostatahyperplasie eingesetzt werden, können bei juxtavesikalen Steinen zu einem reduzierten Schmerzmittelbedarf und einem schnelleren Steinabgang führen [38, 39]. Diese Beobachtungen müssen aber noch durch größere, doppelblind durchgeführte Studien bestätigt werden. Alle Patienten werden aufgefordert, den Urin zu sieben, damit der spontan abgegangene Stein zur Steinanalyse asserviert werden kann.

\section{Interventionelle und operative Steintherapie}

Bei Steinen $>5 \mathrm{~mm}$ oder vergeblichem Warten auf den spontanen Steinabgang müssen interventionelle und endourologische Maßnahmen zur Steinbehandlung in Erwägung gezogen werden. $\mathrm{Zu}$ diesen gehören:

- Extrakorporale Stoßwellen Lithotripsie (ESWL),

- Ureterorenoskopische Steinentfernung (URS)

- percutane Nephrolitholapaxie (PCNL) eines proximalen Harnleitersteines

Operative Behandlungsoptionen im engeren Sinne sind:

- retroperitoneoskopische und laparoskopische Ureterolithotomie

- offene Harnleiterchirurgie

Tabelle 4. Spontane Harnleitersteinabgänge in Abhängigkeit der Steingröße und -lokalisation [33, 34]

\begin{tabular}{lllllll}
\hline Lokalisation & & $\leq 2 \mathrm{~mm}$ & $2-4 \mathrm{~mm}$ & $<5 \mathrm{~mm}$ & $4-6 \mathrm{~mm}$ & $5-10 \mathrm{~mm}$ \\
\hline \multirow{2}{*}{ proximal } & proximal & - & $75 \%$ & & $50 \%$ & $10-53 \%$ \\
distal & Mitte & $77 \%$ & - & $29-98 \%$ & $50 \%$ & $25-53 \%$ \\
& distal & $97 \%$ & $86 \%$ & $71-98 \%$ & $50 \%$ & \\
\hline
\end{tabular}




\section{Extrakorporale Stoßwellenlithotripsie (ESWL)}

Die erstmals 1980 klinisch eingesetzte ESWL hat aufgrund ihrer geringen Invasivität inzwischen eine führende Rolle in der Harnsteinbehandlung eingenommen. Sie kann in Abhängigkeit der Steinzertrümmerungseinheit und der benutzten Stoßwellenstärke meist in Sedoanalgesie durchgeführt werden. Einzige absolute Kontraindikation dieser Therapieform ist eine bestehende Schwangerschaft. Relative Kontraindikationen sind Adipositas, Skelettanomalien, Hypertonie sowie Blutgerinnungsstörungen und antikoagulierte Patienten. Die Behandlungsergebnisse der ESWL von Harnleitersteinen sind insgesamt schlechter als bei Nierensteinen. Ursächlich hierfür ist neben einer erschwerten Ankopplung durch Knochen- oder Darmgasüberlagerung, die geringere Flüssigkeitseinbettung und stärkere Impaktierung des Steins im Ureter. Die Angaben zur Steinfreiheit variieren in der Literatur. Sie liegen nach einer aktuellen Metaanalyse bei 82\% (proximaler Ureter), 73\% (mittlerer Ureter) und 74\% (distaler Ureter) [3]. In einer großen retrospektiven Studie mit über 1500 behandelten Harnleitersteinen betrug die kumulative Steinfreiheit nach der ersten ESWL, der 1., 2. und 3. Re-ESWL 68, 76, 77 resp. 78\% [40]. Führen zwei aufeinanderfolgende ESWL-Behandlungen nicht zum gewünschten Therapieerfolg, ist eine alternative TherapieOption zu wählen, in erster Linie eine URS. Komplikationen einer ESWL-Behandlung (Ureterobstruktion, Infektion, Blutung) sind selten.

\section{Ureterorenoskopie (URS)}

Zur Durchführung einer ureterorenoskopischen Steinextraktion wird eine Spinalanästhesie, bei proximalen Steinen besser eine Vollnarkose benötigt. Heutzutage kommen vermehrt flexible Ureterorenoskope zu Einsatz. Kleinere Konkremente können oft mit einer Fasszange oder einem Körbchen in toto geborgen werden. Größere Konkremente werden mittels Laserlithotripsie (Holmium: YAG-Laser) oder mechanischer Lithotripsie (Ultraschall, pneumatische Lithotripsie) desintegriert. Größere Steindesintegrate sollten entfernt werden, kleinere Steinfragmente können belassen werden, da sie spontan abgehen. Die generell etwas höhere Steinfreiheit nach URS muss gegen die Invasivität dieser Methode abgewogen werden [41-43]. Je proximaler der Stein im Harnleiter liegt, desto invasiver ist ein Entfernungsversuch. Die ESWL ist deshalb bei proximalen Harnleitersteinen $\leq 10 \mathrm{~mm}$ in vielen Kliniken die Therapie der Wahl. Bei proximalen Konkrementen $>10 \mathrm{~mm}$ sind ESWL und URS gleichwertig Therapieoptionen. Die URS bietet bei distalen Harnleitersteinen $\leq 1 \mathrm{~cm}$ im Vergleich zur ESWL die besseren Resultate (Steinfreiheit $89-100 \%$ vs. 45-98\%). Dies geschieht jedoch auch hier auf Kosten der gösseren Invasivität und der damit verbundenen erhöhten Komplikationstrate [44]. Bei Steinen $\geq 10 \mathrm{~mm}$ ist die URS der ESWL noch deutlicher überlegen [45]. Komplikationen treten bei der URS in Abhängigkeit der Steinlokalisation und Erfahrung des Operateurs in 9-11\% der Fälle auf, wobei eine Avulsion der Harnleiterschleimhaut am häufigsten ist. Schwerwiegendere Komplikationen wie Harnleiterabriss oder Ureterperforation sind selten. Gemäß den Empfehlungen der EAU sind sowohl die ESWL als auch die ureterorenoskopische Steinentfernung Therapieoptionen der ersten Wahl bei der Behandlung des Harnleitersteines [3, 29]. Durch die Miniaturisierung der Instrumente und die größere Erfahrung sind die Komplikationen der URS deutlich seltener geworden, so dass sie heute in Anbetracht der signifikant höheren Steinfreiheitsraten als primäre Option insbesondere beim distalen Harnleiterstein anzusehen ist.

\section{Percutane Nephrolitholapaxie (PCNL)}

Die percutane Nephrolitholapaxie wurde initial als minimal invasive Therapiealternative zur offenen Nierensteinchirurgie entwickelt. In den 1980er-Jahren weitgehend von der ESWL verdrängt, erlebt die PCNL seit einigen Jahren eine Renaissance. Grund hierfür sind die mittlerweilen bekannten Grenzen der ESWL. Hierzu zählen Steine der unteren Kelchgruppe $(>1,5 \mathrm{~cm})$, große Steinmasse sowie harte Steinzusammensetzung (Zystin, Kalziumoxalat-Monohydrat) [46]. Als Zugang zum Nierenbeckenhohlsystem dient ein dorsaler Kelch der unteren Kelchgruppe. Der Punktionskanal wird aufgedehnt, so dass eine Zugangshülse ins Nierenbecken vorgeschoben werden kann. Mit verschiedenen Lithotripsie-Sonden und Steinzangen können so nicht nur Steine im Nierenbecken und den Nierenkelchen desintegriert und entfernt werden, sondern auch Steine am pyeloureteralen Übergang und im Bereich des proximalen Harnleiters. Diese antegrade perkutane Steinextraktion aus dem proximalen Harnleiter ist Sonderfällen vorbehalten [3, 29]. Indiziert ist sie z.B. bei gleichzeitig vorhandenen größeren Nierensteinen, bei großen $(>15 \mathrm{~mm})$, impaktierten proximalen Harnleitersteinen sowie bei Patienten mit Harnableitungen (Ileum conduit, Neoblasen) [47, 48]. Bei impaktierten, proximalen Harnleitersteinen sind Steinfreiheitsraten zwischen $85 \%$ und $100 \%$ möglich [49].

\section{Auxiliäre Maßnahmen}

Doppel-J-Einlagen vor ESWL eines Harnleitersteines verbessern das Therapieergebnis nicht und führen $\mathrm{zu}$ einer zusätzlichen Morbidität [33]. Eine Doppel-J-Einlage vor ESWL wird deshalb nicht mehr empfohlen [3]. Ein push back, d.h. das Zurückstoßen eines Harnleitersteines in das Nierenbecken vor ESWL ist mit einer etwas besseren Steindesintegration aber auch mit einer größeren Invasivität als die alleinige ESWL des Steines im Harnleiter assoziiert. Die Manipulation kann sehr schwierig und gelegentlich auch unmöglich sein [29]. Zudem besteht das Risiko, dass sich Steindesintegrate in die untere Kelchgruppe verirren und dort als Residuen verbleiben. Ein push pack wird deshalb nicht mehr empfohlen [3].

Die Einlage eines Doppel-J-Katheters nach URS führt zu signifikanten Schmerzen im Bereich der Niere und der Blase, irritativen Miktionsbeschwerden sowie zu einem höheren Analgetikabedarf. Die Steinfreiheit- und die Strikturrate ca. 7 Wochen postoperativ sind bei Patienten mit und ohne Doppel-J-Katheter gleich. Die Rehospitalisationsrate bei Patienten ohne Stent betrug allerdings bis 7,4\% [50, 51]. Die Einlage einer Harnleiterschiene nach URS ist deshalb nicht obligat und muss individuell intraoperativ entschieden werden. Bei intraoperativen 
Ureterverletzung, Harnleiterstrikturen, anatomischer und funktioneller Einzelniere, Niereninsuffizienz sowie postinterventionell großer residueller Steinlast sollte eine Harnleiterschiene eingelegt werden [3].

Ist eine URS z.B. wegen eines sehr engen Harnleiters nicht durchführbar, erfolgt die Doppel-J-Einlage und 7-14 Tage später ein Doppel-J-Katheter-Auslassversuch (Hoffnung auf spontanen Steinabgang bei kleinen Steinen) oder ein erneuter URS-Versuch.

\section{Retroperitoneoskopische und laparoskopische Ureterolithotomie}

In Zentren mit ausgewiesener laparoskopischer und/ oder retroperitoneoskopischer Erfahrung können Harnleitersteine auch minimal invasiv chirurgisch behandelt werden [3]. Der Harnleiter ist transperitoneal und retroperitoneoskopisch in ganzer Länge zugänglich. Die laparoskopische Ureterolithotomie ist - bei entsprechenden Ressourcen - der offenen Chirurgie aufgrund der bekannten Vorteile der minimal invasiven Chirurgie vorzuziehen. Die Indikation besteht bei sehr großen und impaktierten Harnleitersteinen, sowie bei Therapieversagern der primären Behandlung (ESWL, URS). Studien zeigen vergleichbare Resultate mit der offenen Chirurgie [52, 53].

Die retroperitoneoskopische/laparoskopische Steinextraktion im Bereich des distalen Ureters ist schwieriger als im mittleren und proximalen Abschnitt. Die Größe der Konkremente scheint jedoch keinen Einfluss auf den Therapieerfolg zu besitzen. Obwohl sehr effektiv, ist die retroperitoneoskopische/laparoskopische Steinextraktion keine first-line Therapie des Harnleitersteines. Im Vergleich zur ESWL und URS ist sie invasiver, die Rekonvalesz der Patienten länger und das Risiko schwerwiegender Komplikationen größer.

\section{Offene Steinchirurgie des Harnleiters}

Indikationen zur offenen Steinchirurgie im Bereich des Harnleiters sind selten und auf Einzelfälle beschränkt, bei denen ein minimal-invasives Verfahren inklusive laparoskopische und retroperitoneoskopische Steinentfernung nicht möglich ist. Ziel der offenen Steinchirurgie ist es, den Stein in einer Operation definitiv zu behandeln, resp. zu entfernen. Die dabei zu erwartende Steinfreiheit ist mit $87 \%$ gut [33]. Mögliche Indikationen zur offenen Steinchirurgie sind: anatomische Besonderheiten des Harnleiters, welche eine ESWL/URS unmöglich machen (Adipositas, muskuloskeletale Malformationen, ektope Nieren u.a.). Weiterhin sind Therapieversagen von ESWL und URS, „Risikopatienten“ bei denen wiederholte Eingriffe/Anästhesieverfahren vermieden werden sollen und gleichzeitig stattfindende andere offene Operation zu nennen $[54,55]$.

\section{Zusammenfassung}

Nierensteinleiden und konsekutive Harnleiterkoliken sind häufig. Da meist jüngere berufstätige Männer und Frauen betroffen sind, hat die damit zusammenhängende krankheitsbedingte Arbeitsunfähigkeit auch sozioökonomische Bedeutung.

In der Notfallsituation steht die Schmerz-Behandlung im Vordergrund. Primär kommen nichtsteroidale anti- inflammatorische Agenzien wie Diclofenac und Metamizol, sekundär auch Opiate zum Einsatz.

Basis einer schnellen und effizienten Stein-Behandlung ist eine adäquate Bildgebung, bei der Steinlokalisation und Steingröße zuverlässig erkannt werden können. Empfohlen wird heute primär die native Computertomographie.

Ein Abwarten des spontanen Steinabganges ist bei Steinen $\leq 5 \mathrm{~mm}$ sinnvoll. Rezidivkoliken kann mit Diclofenac vorgebeugt werden. Der spontane Steinabgang, insbesondere von distalen Steinen, wird mit einer alphaBlockertherapie begünstigt.

Ist ein abwartendes Vorgehen nicht möglich oder vom Patienten nicht gewünscht, stehen als primäre Therapieoptionen die ureterorenoskopische Steinentfernung oder die ESWL zur Verfügung. Mit zunehmender Miniaturisierung und Effizienzsteigerung der Ureterorenoskope und Lithotripsiesysteme (insb. Laser) ist die URS mit Steinentfernung/-desintegration in vielen Kliniken zur Therapie der 1. Wahl geworden. Perkutane Steinentfernung über die Niere, laparoskopische und retroperitoneoskopische sowie offen chirurgische Steinentfernungen sind speziellen Indikationen vorbehalten.

Es ist davon auszugehen, dass die Dichte der Computertomographen auch in Zukunft weiter zunehmen wird. Gleichzeitig werden die Untersuchungsprotokolle hinsichtlich Strahlenexposition weiter optimiert werden. Eine weitere Verbesserung und Miniaturisierung der Ureterorenoskope und der ureterorenoskopischen Hilfsinstrumente zur Steinentfernung (Zangen, Körbchen, Lithotriptoren) ist zu erwarten. Aus unserer Erfahrung ist die Bereitschaft der Patienten auf einen Steinabgang zu warten immer geringer und der Anspruch auf eine möglichst schnelle und „Vollständige“ Therapie (Steinentfernung) immer gröBer, sodass davon auszugehen ist, dass die ureterorenoskopissche Harnleitersteinbehandlung in ihrer Bedeutung weiter zunehmen wird.

\section{Literatur}

1. Hesse A, Brandle E, Wilbert D, Kohrmann KU, Alken P (2003) Study on the prevalence and incidence of urolithiasis in germany comparing the years 1979 vs. 2000. Eur Urol 44 (6): 709-713

2. Clark JY, Thomson IM, Optenberg SA (1995) Economic impact of urolithiasis in the united states. J Urol 154 (6): 2020-2024

3. Preminger GM, Tiselius HG, Assimos DG, Alken P, Buck AC, Gallucci M, et al (2007) 2007 Guideline for the management of ureteral calculi. Eur Urol 52 (6): 16101631

4. Press SM, Smith AD (1995) Incidence of negative hematuria in patients with acute urinary lithiasis presenting to the emergency room with flank pain. Urology 45 (5): 753-757

5. Miller OF, Rineer SK, Reichard SR, Buckley RG, Donovan MS, Graham IR, et al (1998) Prospective comparison of unenhanced spiral computed tomography and intravenous urogram in the evaluation of acute flank pain. Urology 52 (6): 982-987

6. Pfister SA, Deckart A, Laschke S, Dellas S, Otto U, Buitrago C, et al (2003) Unenhanced helical computed tomography vs intravenous urography in patients with acute flank pain: accuracy and economic impact in a 
randomized prospective trial. Eur Radiol 13 (11): 25132520

7. Tisdale BE, Siemens DR, Lysack J, Nolan RL, Wilson JW (2007) Correlation of ct scan versus plain radiography for measuring urinary stone dimensions. Can J Urol 14 (2): 3489-3492

8. Heidenreich A, Desgrandschamps F, Terrier F (2002) Modern approach of diagnosis and management of acute flank pain: review of all imaging modalities. Eur Urol 41 (4): $351-362$

9. Ege G, Akman H, Kuzucu K, Yildiz S (2003) Acute ureterolithiasis: incidence of secondary signs on unenhanced helical ct and influence on patient management. Clin Radiol 58 (12): 990-994

10. Poletti PA, Platon A, Rutschmann OT, Schmidlin FR, Iselin CE, Becker CD (2007) Low-dose versus standarddose ct protocol in patients with clinically suspected renal colic. AJR Am J Roentgenol 188 (4): 927-933

11. Eikefjord EN, Thorsen F, Rorvik J (2007) Comparison of effective radiation doses in patients undergoing unenhanced mdct and excretory urography for acute flank pain. AJR Am J Roentgenol 188 (4): 934-939

12. Kluner C, Hein PA, Gralla O, Hein E, Hamm B, Romano V, et al (2006) Does ultra-low-dose ct with a radiation dose equivalent to that of kub suffice to detect renal and ureteral calculi? J Comput Assist Tomogr 30(1): 44-50

13. Mulkens TH, Daineffe S, De Wijngaert R, Bellinck P, Leonard A, Smet G, et al (2007) Urinary stone disease: comparison of standard-dose and low-dose with $4 \mathrm{~d}$ mdct tube current modulation. AJR Am J Roentgenol 188 (2): 553-562

14. Thoeny HC, Tuma J, Hess B (2003) Diagnostic imaging of calculi in the upper urinary tract - sonography vs. computerized tomography. Ther Umsch 60 (2): 73-78

15. Sheafor DH, Hertzberg BS, Freed KS, Carroll BA, Keogan MT, Paulson EK, et al (2000) Nonenhanced helical ct and us in the emergency evaluation of patients with renal colic: prospective comparison. Radiology 217 (3): 792 797

16. Mutgi A, Williams JW, Nettleman M (1991) Renal colic. Utility of the plain abdominal roentgenogram. Arch Intern Med 151 (8): 1589-1592

17. Patlas M, Farkas A, Fisher D, Zaghal I, Hadas-Halpern I (2001) Ultrasound vs ct for the detection of ureteric stones in patients with renal colic. Br J Radiol 74 (886): 901904

18. Mitterberger M, Pinggera GM, Pallwein L, Gradl J, Feuchtner G, Plattner R, et al (2007) Plain abdominal radiography with transabdominal native tissue harmonic imaging ultrasonography vs unenhanced computed tomography in renal colic. BJU Int 100 (4): 887-890

19. Kobayashi T, Nishizawa K, Watanabe J, Ogura K (2003) Clinical characteristics of ureteral calculi detected by nonenhanced computerized tomography after unclear results of plain radiography and ultrasonography. J Urol 170 (3): 799-802

20. Moody TE, Vaughn EDJ, Gillenwater JY (1975) Relationship between renal blood flow and ureteral pressure during 18 hours of total unilateral uretheral occlusion. Implications for changing sites of increased renal resistance. Invest Urol 13 (3): 246-251

21. Travaglini F, Bartoletti R, Gacci M, Rizzo M (2004) Pathophysiology of reno-ureteral colic. Urol Int 72 [Suppl 1]: $20-23$
22. Labrecque M, Dostaler LP, Rousselle R, Nguyen T, Poirier S (1994) Efficacy of nonsteroidal anti-inflammatory drugs in the treatment of acute renal colic. A meta-analysis. Arch Intern Med 154 (12): 1381-1387

23. Holdgate A, Pollock T (2005) Nonsteroidal anti-inflammatory drugs (Nsaids) versus opioids for acute renal colic. Cochrane Database Syst Rev (2): CD004137

24. Collaborative Group of the Spanish Society of Clinical Pharmacology (1991) Comparative study of the efficacy of dipyrone, diclofenac sodium and pethidine in acute renal colic. Eur J Clin Pharmacol 40 (6): 543-546

25. O'Connor A, Schug SA, Cardwell H (2000) A comparison of the efficacy and safety of morphine and pethidine as analgesia for suspected renal colic in the emergency setting. J Accid Emerg Med 17 (4): 261-264

26. Cordell WH, Larson TA, Lingeman JE, Nelson DR, Woods JR, Burns LB, et al (1994) Indomethacin Suppositories Versus Intravenously Titrated Morphine for the Treatment of Ureteral Colic. Ann Emerg Med 23 (2): 262-269

27. Supervia A, Pedro-Botet J, Nogues X, Echarte JL, Minguez S, Iglesias ML, et al (1998) Piroxicam fast-dissolving dosage form vs diclofenac sodium in the treatment of acute renal colic: A Double-Blind Controlled Trial. Br J Urol 81 (1): 27-30

28. Nissen I, Birke H, Olsen JB, Wurtz E, Lorentzen K, Salomon H, et al (1990) Treatment of ureteric colic. Intravenous versus rectal administration of indomethacin. $\mathrm{Br} \mathrm{J}$ Urol 65 (6): 576-579

29. Tiselius HG, Ackermann D, Alken P, Buck C, Conort P, Gallucci M (2001) Guidelines on urolithiasis. Eur Urol 40 (4): 362-371

30. Pearle MS, Pierce HL, Miller GL, Summa JA, Mutz JM, Petty BA, et al (1998) Optimal method of urgent decompression of the collecting system for obstruction and infection due to ureteral calculi. J Urol 160 (4): 1260-1264

31. Mokhmalji H, Braun PM, Martinez Portillo FJ, Siegsmund M, Alken P, Kohrmann KU (2001) Percutaneous nephrostomy versus ureteral stents for diversion of hydronephrosis caused by stones: a prospective, randomized clinical trial. J Urol 165 (4): 1088-1092

32. Joshi HB, Obadeyi OO, Rao PN (1999) A comparative analysis of nephrostomy, JJ stent and urgent in situ extracorporeal shock wave lithotripsy for obstructing ureteric stones. BJU Int 84 (3): 264-269

33. Segura JW, Preminger GM, Assimos DG, Dretler SP, Kahn RI, Lingeman JE, et al (1997) Ureteral stones clinical guidelines panel summary report on the management of ureteral calculi. The American Urological Association. J Urol 158 (5): 1915-1921

34. Miller OF, Kane CJ (1999) Time to stone passage for observed ureteral calculi: a guide for patient education. J Urol 162 (3 Pt 1): 688-90; discussion 690-691

35. Holm-Nielsen A, Jorgensen T, Mogensen P, Fogh J (1981) The prognostic value of probe renography in ureteric stone obstruction. Br J Urol 53 (6): 504-507

36. Irving SO, Calleja R, Lee F, Bullock KN, Wraight $\mathrm{P}$, Doble A (2000) Is the conservative management of ureteric calculi of $>4 \mathrm{~mm}$ safe? BJU Int 85 (6): 637-640

37. Laerum E, Ommundsen OE, Gronseth JE, Christiansen A, Fagertun HE (1995) Oral diclofenac in the prophylactic treatment of recurrent renal colic. A double-blind comparison with placebo. Eur Urol 28 (2): 108-111

38. Hollingsworth JM, Rogers MA, Kaufman SR, Bradford TJ, Saint S, Wei JT, et al (2006) Medical therapy to fa- 
cilitate urinary stone passage: a meta-analysis. Lancet 368 (9542): 1171-1179

39. Parsons JK, Hergan LA, Sakamoto K, Lakin C (2007) Efficacy of alpha-blockers for the treatment of ureteral stones. J Urol 177 (3): 983-987; discussion 987

40. Pace KT, Weir MJ, Tariq N, Honey RJ (2000) Low success rate of repeat shock wave lithotripsy for ureteral stones after failed initial treatment. J Urol 164 (6): 19051907

41. Strohmaier WL, Schubert G, Rosenkranz T, Weigl A (1999) Comparison of extracorporeal shock wave lithotripsy and ureteroscopy in the treatment of ureteral calculi: A prospective study. Eur Urol 36 (5): 376-379

42. Kupeli B, Biri H, Isen K, Onaran M, Alkibay T, Karaoglan U, et al (1998) Treatment of ureteral stones: Comparison of extracorporeal shock wave lithotripsy and endourologic alternatives. Eur Urol 34 (6): 474-479

43. Nabi G, Downey P, Keeley F, Watson G, McClinton S (2007) Extra-corporeal shock wave lithotripsy (eswl) versus ureteroscopic management for ureteric calculi. Cochrane Database Syst Rev (1): CD006029

44. Lam JS, Greene TD, Gupta M (2002) Treatment of proximal ureteral calculi: Holmium: Yag laser ureterolithotripsy versus extracorporeal shock wave lithotripsy. J Urol 167 (5): 1972-1976

45. Kupeli B, Alkibay T, Sinik Z, Karaoglan U, Bozkirli I (2000) What is the optimal treatment for lower ureteral stones larger than $1 \mathrm{~cm}$ ? Int J Urol 7 (5): 167-171

46. Knoll T, Wendt-Nordahl G, Alken P (2005) Clinical value of percutaneous nephrolithotomy. Urologe A 44 (3): 299306; quiz 307-308

47. Kumar V, Ahlawat R, Banjeree GK, Bhaduria RP, Elhence A, Bhandari M (1996) Percutaneous ureterolitholapaxy: the best bet to clear large bulk impacted upper ureteral calculi. Arch Esp Urol 49 (1): 86-91

48. Goel R, Aron M, Kesarwani PK, Dogra PN, Hemal AK, Gupta NP (2005) Percutaneous antegrade removal of im- pacted upper-ureteral calculi: still the treatment of choice in developing countries. J Endourol 19 (1): 54-57

49. Karami H, Arbab AH, Hosseini SJ, Razzaghi MR, Simaei NR (2006) Impacted upper-ureteral calculi $>1 \mathrm{~cm}$ : blind access and totally tubeless percutaneous antegrade removal or retrograde approach? J Endourol 20 (9): 616619

50. Borboroglu PG, Amling CL, Schenkman NS, Monga M, Ward JF, Piper NY, et al (2001) Ureteral stenting after ureteroscopy for distal ureteral calculi: a multi-institutional prospective randomized controlled study assessing pain, outcomes and complications. J Urol 166 (5): 16511657

51. Denstedt JD, Wollin TA, Sofer M, Nott L, Weir M, D‘A Honey RJ (2001) A prospective randomized controlled trial comparing nonstented versus stented ureteroscopic lithotripsy. J Urol 165 (5): 1419-1422

52. Skrepetis K, Doumas K, Siafakas I, Lykourinas M (2001) Laparoscopic versus open ureterolithotomy. a comparative study. Eur Urol 40 (1): 32-36; discussion 37

53. Goel A, Hemal AK (2001) Upper and mid-ureteric stones: a prospective unrandomized comparison of retroperitoneoscopic and open ureterolithotomy. BJU Int 88 (7): 679-682

54. Ather MH, Paryani J, Memon A, Sulaiman MN (2001) A 10 -year experience of managing ureteric calculi: changing trends towards endourological intervention - is there a role for open surgery? BJU Int 88 (3): 173-177

55. Muslumanoglu AY, Karadag MA, Tefekli AH, Altunrende F, Tok A, Berberoglu Y (2006) When is open ureterolithotomy indicated for the treatment of ureteral stones? Int $\mathrm{J}$ Urol 13 (11): 1385-1388

Korrespondenz: Dr. med. Thomas H. Forster, Urologische Klinik Solothurner Spitäler AG (soH), Baslerstrasse 150, 4600 Olten, Schweiz, E-Mail: tforster_ol@spital.ktso.ch. 